\title{
OBSTRUCTIONS TO EMBEDDING AND ISOTOPY IN THE METASTABLE RANGE
}

\author{
LAWRENCE L. LARMORE
}

\section{Introduction.}

1.1. Preliminary definitions and summary. Throughout this paper, "manifold" means differentiable manifold (closed or open) without boundary, with a countable base. "Differentiable" means infinitely differentiable, and "embedding" means differentiable embedding.

Suppose $V$ and $M$ are manifolds of dimension $k$ and $n$, respectively, $V$ compact, and $f: V \rightarrow M$ is a differentiable map. An embedding homotopy of $f$ (abbreviated $e$-homotopy) shall be defined to be a homotopy of differentiable maps, $f_{t}: V \rightarrow M$, for $0 \leqq t \leqq 1$, such that $f_{0}=f$ and $f_{1}$ is an embedding. We say that $e$-homotopies $\left\{f_{0, t}\right\}$ and $\left\{f_{1, t}\right\}$ are isotopic if there exists a 2-parameter homotopy of differentiable maps $f_{\tau, t}: V \rightarrow M$, for $0 \leqq \tau, t \leqq 1$, such that $f_{\tau, 0}=f$ and $f_{\tau, 1}$ is an embedding for all $\tau$. Let $\left[f_{t}\right]$ denote the isotopy class of $\left\{f_{t}\right\}$, and let $[V \subset M]_{f}$ denote the set of all isotopy classes of $e$ homotopies of $f$.

It is not difficult to show that if $f$ is an embedding, $[V \subset M]_{f}$ naturally has the structure of an Abelian group with identity $[f]$ (where $\{f\}$ is the constant homotopy), provided $2 n>3(k+1)$. However, this construction is not within the scope of the present paper; we refer the reader to J. C. Becker [1] for the case when $M$ is a Euclidean space. [ $\left.V \subset R^{n}\right]_{f}$ becomes $E(V, n)$, the so-called embedding group.

We consider three problems in this paper. The first is existence of an $e$-homotopy of $f$, i.e., whether $[V \subset M]_{f}$ is nonempty; the second is enumeration of $[V \subset M]_{f}$; more precisely, whether two given $e$ homotopies are isotopic. The third question deals with the function $\Delta:[V \subset M]_{f} \rightarrow[V \subset M]$, where $[V \subset M]$ is the set of isotopy classes of embeddings of $V$ into $M$, and where, for any $e$-homotopy $\left\{f_{t}\right\}$ of $f$, $\Delta\left[f_{t}\right]=\left[f_{1}\right]$, the isotopy class containing $f_{1}$. As we see in $\$ 3.5$, there is an action of $\pi_{1}\left(M^{V}, f\right)$ on $[V \subset M]_{f}$ whose orbits correspond to the image of $\Delta$, where $M^{V}$ is the space of differentiable functions $V \rightarrow M$ with the compact-open topology. In $\S 3.8$, we discuss

Received by the editors December 19, 1970.

AMS (MOS) subject classifications (1970). Primary 57O40, 57G35; Secondary 57B30. 
that action in the special case that $k \geqq 2, V=S^{k}$, the $k$-sphere, $n=$ $2 k+1$, and $f$ is inessential.

We translate the problem of existence and isotopy of $e$-homotopies of $f$ into a lifting problem, using Haefliger's results [4]. In \$3.1, we define a $2 k$-manifold $R^{*} V$ with boundary $P V$, the total space of the projective bundle associated with the tangent bundle of $V . R^{*} V$ has the homotopy type of the reduced deleted product of $V,\left(V^{2}-\Delta_{V}\right) / T$, where $\Delta_{V}$ is the diagonal and $T$ exchanges coordinates. In $\S 3.2$ we define a pair of spaces $\left(Y^{\prime}, Z^{\prime}\right)$ and a map $\pi_{M}{ }^{\prime}:\left(Y^{\prime}, Z^{\prime}\right) \rightarrow\left(R^{*} V, P V\right)$ such that $\pi_{M}{ }^{\prime}$ and $\pi_{M}{ }^{\prime} \mid Z^{\prime}$ are both fibrations, and for each $e$-homotopy $\left\{f_{t}\right\}$ of $f$ we define a specific section of $\pi_{M}{ }^{\prime}, \Phi\left[f_{t}\right]$ : $\left(\boldsymbol{R}^{*} V, P V\right) \rightarrow\left(Y^{\prime}, Z^{\prime}\right)$. The function $\phi:[M \subset V]_{f} \rightarrow \operatorname{Sec}\left(\pi_{M}{ }^{\prime}\right)$ which sends each $\left[f_{t}\right]$ to $\left[\boldsymbol{\Phi}\left[f_{t}\right]\right]$, the class containing $\boldsymbol{\Phi}\left[f_{t}\right]$ (where $\operatorname{Sec}\left(\pi_{M}{ }^{\prime}\right)$ is the set of homotopy classes of sections of $\pi_{M}{ }^{\prime}$, two sections being homotopic if they are connected by a homotopy of sections) is onto if $2 n \geqq 3(k+1)$ and one-to-one if $2 n>3(k+1)$ (see Theorems 3.3.1 and 3.3.2). The obstruction theory for sections of fibrations of pairs, developed in $\$ 2$, can then be applied.

The first obstruction to finding an $e$-homotopy of $f$ lies in $H^{n}\left(\boldsymbol{R}^{*} V ; \pi_{n-1}\right)$, and higher obstructions lie in $H^{n+i}\left(\boldsymbol{R}^{*} V ; \pi_{n+i-1}\right)$ for $i \geqq 1$, where $\pi_{n+i-1}$ is a sheaf of Abelian groups over $\boldsymbol{R}^{*} V$ which is not generally even locally a product sheaf, for $i \geqq 0$. (When restricted to either $P V$ or $R^{*} V-P V$, however, $\pi_{n+i-1}$ is locally trivial, i.e., locally a product sheaf.) The first obstruction to isotopy of two $e$-homotopies of $f$ lies in $H^{n-1}\left(\boldsymbol{R}^{*} V ; \pi_{n-1}\right)$; higher obstructions lie in $H^{n+i-1}\left(\boldsymbol{R}^{*} V ; \pi_{n+i-1}\right)$ for $i \geqq 1$.

Thus (cf. Theorems 2.5.1 and 3.3.2), [ $V \subset M]_{f}$ is in one-to-one correspondence with $H^{2 k}\left(\boldsymbol{R}^{*} V ; \pi_{2 k}\right)$ if $k \geqq 2$ and $n=2 k+1$. This correspondence is canonical if $f$ is an embedding; $[f]$ then corresponds to 0 . Identifying the two sets in that case, we then say that $[V \subset M]_{f}$ is an Abelian group.

1.2. Applications. Suppose now that $V=S^{k}$, for $k \geqq 2$, and $n=$ $2 k+1$. Let $x \in M$ be a basepoint, and let $f: S^{k} \rightarrow M$ be a basepoint-preserving embedding. Define $d: \pi_{1}(M, x) \rightarrow Z_{2}$ to be the orientation homomorphism, i.e., the kernel of $d$ is the image of the fundamental group of the orientation covering space of $M$.

TheOREM 1.2.1. $\left[S^{k} \subset M\right]_{f}$ is generated by elements $\langle g\rangle$ for all $g \in \pi_{1}(M, x)$, subject only to the following relations:

(i) $\langle 1\rangle=0$, where 1 is the identity of $\pi_{1}(M, x)$.

(ii) $\left\langle g^{-1}\right\rangle=(-1)^{k+1}(-1)^{d(g)}\langle g\rangle$ for all $g \in \pi_{1}(M, x)$.

The reader can easily verify that if $\pi_{k+1}(M, x)=0$, the evaluation on 
the basepoint of $S^{k}, M^{s^{k}} \rightarrow M$, induces an isomorphism $\pi_{1}\left(M^{s^{k}}, f\right)$ $\rightarrow \pi_{1}(M, x)$, provided $f$ is inessential; we identify these groups for convenience.

THeOREM 1.2.2. Suppose $f$ is small, i.e., $f\left(S^{k}\right)$ is contained in a single chart of $M$, and $\pi_{k+1}(M)=0$. The action of $\pi_{1}\langle M, x)$ on $\left[S^{k} \subset M\right]_{f}$ is given by $(\langle g\rangle, h) \mapsto \rightarrow(-1)^{d(h)}\left\langle h^{-1} g h\right\rangle$ for all $g$, $h \in \pi_{1}(M, x)$.

In the following applications, 0 will be a small embedding, $\pi_{1}$ will be the fundamental group of the space into which we are embedding $S^{k}$.

THEOREM 1.2.3 (HACON [3]). For $k \geqq 2,\left[S^{k} \subset S^{1} \times S^{2 k}\right]_{0}$ is isomorphic to the direct sum of countably many copies of the integers, and the action of $\pi_{1} \cong \mathrm{Z}$ is trivial.

Suppose now that $k \geqq 2$, and $P_{r}$ is a real projective $r$-space, for $k+2 \leqq r \leqq 2 k+1$. Let $G=\left[S^{k} \subset P_{r} \times R^{2 k-r+1}\right]_{0}$.

Theorem 1.2.4. Case I. If $k$ and $r$ are even, $G \cong Z_{2}$ and the action of $\pi_{1}$ is trivial. Case II. If $k$ and $r$ are both odd, $G \cong Z_{2}$ and the action of $\pi_{1}$ is trivial. Case III. If $k$ is even and $r$ is odd, $G \cong Z$ and the action of $\pi_{1}$ is trivial. Case IV. If $k$ is odd and $r$ is even, $G \cong Z$ and the action of $\pi_{1} \cong Z_{2}$ is nontrivial; the generator of $\pi_{1}$ takes every element of $G$ to its inverse.

Theorems 1.2.3 and 1.2.4 follow immediately from 1.2.1 and 1.2.2, as the reader may easily verify.

1.3. Embeddings in a Euclidean space. Let $V$ be a compact $k$ dimensional manifold, as before, and let $M=R^{n}$. Our obstruction theory then reduces to a simpler theory. The first obstruction to embedding $V$ in $R^{n}$ lies in $H^{n}\left(R^{*} V ; Z\right)$, where $\mathcal{Z}=Z$ if $n$ is even and $\mathcal{Z}$ is the twisted integer sheaf (sometimes called $Z^{T}$ ) if $n$ is odd. Higher obstructions lie in $H^{n+i}\left(\boldsymbol{R}^{*} V ; \mathcal{Z} \otimes \pi_{i}\right)$ for $i \geqq 1$, where $\pi_{i}$ is the stable $i$-stem in the homotopy of spheres. The first obstruction to isotopy of two embeddings lies in $H^{n-1}\left(\boldsymbol{R}^{*} V ; \mathcal{Z}\right)$; higher obstructions lie in $H^{n+i-1}\left(\boldsymbol{R}^{*} V ; \mathcal{Z} \otimes \pi_{i}\right)$ for $i \geqq 1 . V$ embeds in $R^{n}$ if and only if all obstructions vanish, provided $2 n \geqq 3(k+1)$; two embeddings are isotopic if and only if all obstructions vanish, provided $2 n>3(k+1)$ [7], [8].

2. Fibrations of pairs.

2.1. Preliminary definitions. Throughout this section, we let $(K, L)$ be an oriented simplicial pair, $(Y, Z)$ another pair of spaces, and $\pi:(Y, Z) \rightarrow(K, L)$ a map of pairs such that $\pi$ and $\pi \mid Z$ are both fibra- 
tions. We say that $\pi$ is a fibration of pairs over $(K, L)$. If $c:(K, L) \rightarrow$ $(Y, Z)$ is a map of pairs such that $\pi \circ c$ is the identity on $K$, we say that $c$ is a section of $\pi$. We say that two sections of $\pi, c_{0}$ and $c_{1}$, are homotopic if there is a homotopy $c_{t}$, for $0 \leqq t \leqq 1$, of sections of $\pi$. For each cell $\sigma \subset K$, let $E_{\sigma}=\pi^{-1} \sigma$ if $\sigma \not L$, and let $E_{\sigma}=$ $\pi^{-1} \sigma \cap Z$ if $\sigma \subset L$. Let $\pi_{\sigma}: E_{\sigma} \rightarrow \sigma$ be the restriction of $\pi$ to $E_{\sigma}$.

We can immediately pose two questions. First: When does $\pi$ have a section; and second: When are two sections homotopic? Suppose $A$ is a subcomplex of $K$, and $h$ is a section of $\pi$ over $A$, i.e., a map of pairs $h:(K \cap A, L \cap A) \rightarrow(Y, Z)$ such that $\pi \circ h$ is the identity. The relative versions of our questions are: When can $h$ be extended over $K$, and when are two such extensions homotopic rel $A$ ?

2.2. The sheaf of homotopy groups. We shall define a sheaf $\pi_{n}=$ $\pi_{n}(\pi)=(\mathcal{G}, p)$ over the space $K$, which we call the sheaf of $n$th homotopy groups of $\pi$ for any $n \geqq 1$, provided that $E_{\sigma}$ is $n$-simple for all cells $\sigma$. As a set, $\mathcal{G}$ will be defined to be the union, over all cells $\sigma \subset K$, of Int $\sigma \times \pi_{n}(E)$; and $p(x, a)=a$ for all $\sigma$, all $x \in$ Int $\sigma$, and all $a \in \pi_{n}\left(E_{\sigma}\right)$. The stalk of $\pi_{n}$ over $x$ we identify with $\pi_{n}\left(E_{\sigma}\right)$. In order to describe the topology of $\mathcal{G}$ it is only necessary to describe continuous sections over open stars of cells, where, if $\sigma$ is a cell of $K$, St $\sigma$, the open star of $\sigma$, is the union of the interiors of all cells of which $\sigma$ is a face. We then say that a section $f:$ St $\sigma \rightarrow \mathcal{G}$ is continuous if for any cell $\tau \supset \sigma$ and any $x \in \operatorname{Int} \tau, f(x)=\left(x, i_{\#} a_{0}\right)$, where $i: E_{\sigma} \rightarrow E_{\sigma}$ is the inclusion, $x_{0}$ is the barycenter of $\sigma$, and $a_{0}=f\left(x_{0}\right)$. We can thus immediately identify the group of continuous sections of $\mathcal{G}$ over St $\sigma$ with $\pi_{n}\left(E_{\sigma}\right)$, by evaluating each section at $x_{0}$.

For any subcomplex $A \subset K$ (not necessarily $L$ ) let $C^{*}=$ $\left(C^{*}\left(K, A ; \pi_{n}\right), \delta\right)$ be the graded differential complex of degree 1 where $C^{k}\left(K, A ; \pi_{n}\right)$ is defined to be the set of all $k$-cochains, i.e., functions $c$ whose domain is the set of $k$-cells of $K$, where $c(\sigma) \in \pi_{n}\left(E_{\sigma}\right)$ for each $k$-cell $\sigma$, and where $c(\sigma)=0$ if $\sigma \subset A$. If $c \in C^{k}\left(K, A ; \pi_{n}\right)$ is any $k$-cochain, we define $\delta c \in C^{k+1}\left(K, A ; \pi_{n}\right)$ as follows: For any $(k+1)$-cell $\tau$, let $\delta c(\tau)=\sum[\sigma ; \tau] c(\boldsymbol{\sigma})$, where the sum is over all $k$-cells $\sigma \subset \tau$, and where $[\sigma ; \tau]= \pm 1$ is the incidence number. According to Theorem 5.1 of [6], we may identify $H^{*}\left(K, A ; \pi_{n}\right)$ with the homology of the graded complex $C^{*}$. If $A$ is empty, we write $C^{k}\left(K ; \pi_{n}\right)$ for $C^{k}\left(K, \varnothing ; \pi_{n}\right)$, etc.

2.3. The obstruction cochain. Let $f:\left(\bar{K}^{n}, \bar{L}^{n}\right) \rightarrow(Y, Z)$ be a section of $\pi$ over $\bar{K}^{n}=K^{n} \cup A$, where $K^{n}$ is the $n$-skeleton of $K$. We consider the question of extension of $f$ to the $(n+1)$-skeleton. Let $c^{n+1}=c^{n+1}(f)$, an element of $C^{n+1}\left(K, A ; \pi_{n}\right)$, be defined as fol- 
lows: If $\sigma \subset K$ is an $(n+1)$-cell and if $\phi: S^{n} \rightarrow \partial \sigma$ is the standard homeomorphism (whose degree is determined by the orientation of $\sigma)$, we let $c^{n+1}(\sigma) \in \pi_{n}\left(E_{\sigma}\right)$ be the homotopy class represented by the composition $f \circ \phi: S^{n} \rightarrow E_{\sigma}$. As in the usual obstruction setting, we have some theorems, which we state without proof (see Hu [5, Chapter 6]).

THEOREM 2.3.1. The obstruction cochain $c^{n+1}(f)$ is an invariant of the homotopy class of $f \operatorname{rel} A$, i.e., if $f_{t}:\left(\bar{K}^{n}, \bar{L}^{n}\right) \rightarrow(Y, Z)$, for $0 \leqq t \leqq 1$, is a homotopy of extensions of $h$, then $c^{n+1}\left(f_{0}\right)=$ $c^{n+1}\left(f_{1}\right)$.

THEOREM 2.3.2. An extension of $f$ over $\bar{K}^{n+1}$ exists if and only if $c^{n+1}(f)=0$.

THEOREM 2.3.3. $c^{n+1}(f)$ is a cocycle.

We may thus define $\gamma^{n+1}(f) \in H^{n+1}\left(K, A ; \pi_{n}\right)$ to be the cohomology class of $c^{n+1}(f)$.

THEOREM 2.3.4. $\gamma^{n+1}(f)=0$ if and only if $f \mid \bar{K}^{n-1}$ can be extended to $\bar{K}^{n+1}$.

2.4. The difference cochain. Suppose $f_{0}$ and $f_{1}$ are sections of $\pi$ over $\overline{\boldsymbol{K}}^{n}$ which are extensions of $h$, and that $g_{t}$, for $0 \leqq t \leqq 1$, is a homotopy of extensions of $h$ over $\bar{K}^{n-1}, g_{i}=f_{i} \mid \bar{K}^{n-1}$ for $i=0,1$. Let $d^{n}=d^{n}\left(f_{0}, f_{1} ; g_{t}\right) \in C^{n}\left(K, A ; \pi_{n}\right)$ be defined as follows.

Let $\pi \times 1:(Y \times I, Z \times I) \rightarrow(K \times I, L \times I)$ be the obvious fibration pair. We define a section $F$ of $\pi \times 1$ over $\bar{K}^{n} \times \partial I \cup \bar{K}^{n-1} \times I$ as follows:

$$
F(x, t)= \begin{cases}\left(f_{t}(x), t\right) & \text { if } t=0 \text { or } 1, \text { for all } x \in \bar{K}^{n}, \\ \left(g_{t}(x), t\right) & \text { if } x \in \bar{K}^{n-1}, \text { for all } t \in I .\end{cases}
$$

Now $c^{n+1}(F) \in C^{n+1}\left(K \times I, A \times I \cup K \times \partial I ; \pi_{n}(\pi \times 1)\right)$. But that group is isomorphic in an obvious way to $C^{n}\left(K, A ; \pi_{n}\right)$, since $\pi_{n}(\pi \times 1)$ $=p^{-1} \pi_{n}$, where $p: K \times I \rightarrow K$ is the projection. Let $d^{n}$ be the image of $c^{n+1}(F)$ under that isomorphism. We state without proof analogues of the usual theorems on difference cochains.

THEOREM 2.4.1. $d^{n}\left(f_{0}, f_{1} ; g_{t}\right)$ is a homotopy invariant.

THEOREM 2.4.2. $\left\{g_{t}\right\}$ can be extended to a homotopy of $f_{0}$ with $f_{1}$ if and only if $d^{n}\left(f_{0}, f_{1} ; g_{t}\right)=0$.

TheOrem 2.4.3. $\delta d^{n}\left(f_{0}, f_{1} ; g_{t}\right)=c^{n+1}\left(f_{1}\right)-c^{n+1}\left(f_{0}\right)$.

Thus, if $f_{0}$ and $f_{1}$ can both be extended to $\bar{K}^{n+1}, d^{n}\left(f_{0}, f_{1} ; g_{t}\right)$ 
is a cocycle; let $\delta^{n}\left(f_{0}, f_{1} ; g_{t}\right) \in H^{n}\left(K, A ; \pi_{n}\right)$ be its cohomology class.

THEOREM 2.4.4. If $k_{t}, 0 \leqq t \leqq 1$, is a homotopy of $f_{1} \mid \bar{K}^{n-1}$ with $f_{2} \mid \bar{K}^{n-1}$, where $f_{2}$ is another extension of $h$ over $\bar{K}^{n}$, then $d^{n}\left(f_{0}, f_{2} ; r_{t}\right)=d^{n}\left(f_{0}, f_{1} ; g_{t}\right)+d^{n}\left(f_{1}, f_{2} ; k_{t}\right)$, where $r_{t}=g_{2 t}$ if $0 \leqq t \leqq \frac{1}{2}$, $r_{t}=k_{2 t-1}$ if $\frac{1}{2} \leqq t \leqq 1$.

TheOREM 2.4.5. If $f_{0}$ and $f_{1}$ can both be extended to $\bar{K}^{n+1}$, then $g_{t} \mid \bar{K}^{n-2}$ can be extended to a homotopy of $f_{0}$ with $f_{1}$ if and only if $\delta^{n}\left(f_{0}, f_{1} ; g_{t}\right)=0$.

THEOREM 2.4.6. For any $f_{0}$ and any homotopy $g_{t}$, as before, and, for any $d \in C^{n}\left(K, A ; \pi_{n}\right)$, there exists an extension $f_{1}{ }^{\prime}$ of $g_{1}$ such that $d^{n}\left(f_{0}, f_{1}{ }^{\prime} ; g_{t}\right)=d$.

2.5. A classification theorem. Suppose that $\pi$ is $(n-1)$-connected, i.e., each $E_{\sigma}$ is connected, and $\pi_{k}=0$ for all $k<n$, for some integer $n \geqq 1$. Suppose also that $\operatorname{dim} K \leqq n$. Let $[K, h ; \pi]$ be the set of rel $A$ homotopy classes of extensions of $h$ over $K$. (If $A$ is empty, write $[K ; \pi]$.

TheOREM 2.5.1. [ $K, h ; \pi]$ can be put into one-to-one correspondence with $H^{n}\left(K, A ; \pi_{n}\right)$.

Proof. By successive application of Theorem 2.3.2 on the skeleta of $K$, we can choose a section $f_{0}$ of $\pi$ such that $f_{0} \mid A=h$. Now let $f$ be any other extension of $h$ over $K$. By Theorem 2.4.2, $f_{0} \mid \bar{K}^{n-1}$ and $f \mid \bar{K}^{n-1}$ are homotopic rel $A$. Pick a homotopy $\left\{g_{t}\right\}$. Let $[f] \in[K, h ; \pi]$, the homotopy class of $f$, correspond to the difference cohomology class $\delta^{n}\left(f_{0}, f ; g_{t}\right)$. By Theorems 2.4.1, 2.4.2, and 2.4.3, this correspondence is well defined; by Theorem 2.4 .5 it is one-to-one, and by 2.4.6 it is onto.

\section{Existence of embeddings and isotopies.}

3.1. The space $R^{*} M$. Let $M$ be any $n$-dimensional manifold, for any integer $n$. Let $S M$ and $P M$ be the total spaces of the sphere bundle and the projective bundle, respectively, associated to the tangent bundle of $M$. Let $R M=M^{2}-\Delta_{M}$, the deleted product of $M$, and let $R^{*} M=R M / T$, where $T$ is the map which exchanges coordinates. We call $R^{*} M$ the reduced deleted product of $M$. Let $\phi: R M \cup S M \rightarrow$ $R^{N} \times R^{N} \times S^{N-1}$ be the map where

$$
\phi(x, y)=(g x, g y,(g x-g y) /\|g x-g y\|)
$$

for all $(x, y) \in R M$, and $\phi(v)=\left(g \pi v, g \pi v, g_{*} v\left\|g_{*} v\right\|^{-1}\right)$ for any unit 
tangent vector $v \in S M$, where $g: M \rightarrow R^{N}$ is any distal embedding of $M$ in any Euclidean space, and $\pi: S M \rightarrow M$ is the projection. Let $\boldsymbol{R} M$ be the topological space $(R M \cup S M, \mathcal{J})$, where $\square$ is the unique topology which makes $\phi$ an embedding. $R M$ also has the structure of a differentiable manifold with boundary $S M$; we leave verification to the reader. Let $T: S M \rightarrow S M$ also denote the antipodal map on each fiber of $\pi ; T$ then acts continuously on $R M$; we define $R^{*} M$ to be the quotient space $R M / T$, also a $2 n$-manifold, with boundary $P M$. We remark that $\boldsymbol{R} M$ and $\boldsymbol{R}^{*} \boldsymbol{M}$ have the same homotopy types as $R M$ and $R^{*} M$, respectively, since if we remove the boundary of any manifold, it does not change the homotopy type.

If $V$ is another manifold and if $f: V \rightarrow M$ is an embedding, maps $R f: R V \rightarrow R M, R^{*} f: R^{*} V \rightarrow R^{*} M, \boldsymbol{R} f: \boldsymbol{R} V \rightarrow \boldsymbol{R} M$, and $\boldsymbol{R}^{*} f: \boldsymbol{R}^{*} V$ $\rightarrow R^{*} M$ are naturally defined. $R f(x, y)=(f x, f y)$, etc.

Let $R^{\infty}=$ the union of $R^{N}$, for all $N \geqq 1$, with the weak topology. We then define $R\left(M \times R^{\infty}\right), R^{*}\left(M \times R^{\infty}\right), S\left(M \times R^{\infty}\right), P\left(M \times R^{\infty}\right)$, $\boldsymbol{R}\left(M \times R^{\infty}\right)$, and $\boldsymbol{R}^{*}\left(M \times R^{\infty}\right)$, to be the unions of the corresponding constructions on $M \times R^{N}$, over all integers $N \geqq 1$, with the weak topology.

3.2. The obstructions to embedding and isotopy. Let $M$ be an $n$-dimensional manifold. We replace the inclusion of pairs $\left(R^{*} M, P M\right)$ $\subset\left(R^{*}\left(M \times R^{\infty}\right), P\left(M \times R^{\infty}\right)\right)$ with a fibration of pairs $\pi_{M}:(Y, Z) \rightarrow$ $\left(R^{*}\left(M \times R^{\infty}\right), P\left(M \times R^{\infty}\right)\right)$ of the same homotopy type. Specifically, let $Y=\left\{\alpha \in R^{*}\left(M \times R^{\infty}\right)^{I} \mid \alpha(1) \in R^{*} M\right\}$, and

$$
Z=\left\{\alpha \in Y \mid \alpha(t) \in P\left(M \times R^{\infty}\right), \text { all } t\right\},
$$

where $R^{*}\left(M \times R^{\infty}\right)^{I}$ is the space of all paths in $R^{*}\left(M \times R^{\infty}\right)$ with the compact-open topology. We let $\pi_{M}(\alpha)=\alpha(0)$ for all $\alpha \in Y$.

Let $V$ be a compact manifold of dimension $k$, and $f: V \rightarrow M$ a differentiable map. Choose, once and for all, an embedding $i: V \rightarrow R^{\infty}$. Let $\left(Y^{\prime}, Z^{\prime}\right)$ be the pullback, as in the diagram:

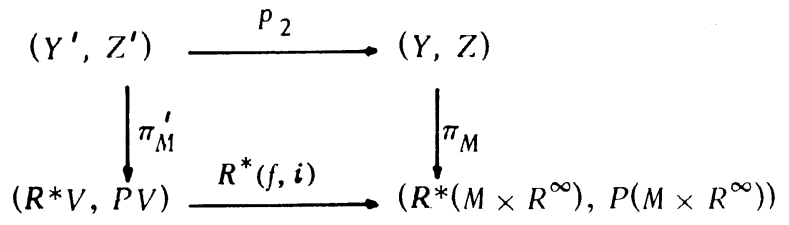

Specifically, we let $Y^{\prime}=\left\{(r, \alpha) \in R^{*} V \times Y \mid R^{*}(f, i)(r)=\alpha(0)\right\}$, and $Z^{\prime}=Y^{\prime} \cap P V \times Z ; \quad \pi_{M}{ }^{\prime}(r, \alpha)=r \quad$ and $p_{2}(r, \alpha)=\alpha$ for all $(r, \boldsymbol{\alpha}) \in Y^{\prime}$.

Now if $f$ is homotopic to an embedding, $\pi_{M}{ }^{\prime}$ has a section; specifically, if $\left\{f_{t}\right\}$ is an $e$-homotopy of $f$, let $\Phi\left[f_{t}\right](r)=(r, \alpha) \in Y^{\prime}$ 
for all $r \in R^{*} V$, where, for any $0 \leqq t \leqq 1, \alpha(t)=R^{*}\left(f_{2 t}, i\right)(r)$ if $0 \leqq t \leqq \frac{1}{2}$, $\boldsymbol{\alpha}(t)=\boldsymbol{R}^{*}\left(f_{1},(2-2 t)\right) \boldsymbol{i}(r)$ if $\frac{1}{2} \leqq \mathrm{t} \leqq 1$. If $\left\{f_{t}\right\}$ and $\left\{g_{t}\right\}$ are $e^{-}$ homotopies of $f$ which are isotopic, $\Phi\left[f_{t}\right]$ and $\Phi\left[g_{t}\right]$ are homotopic as sections of $\pi_{M}{ }^{\prime}$. The converses of these two statements are true in a suitable metastable range, as we shall see in the next paragraph; the obstructions to finding a section of $\pi_{M}{ }^{\prime}$, and to finding a homotopy of two sections, as defined in $\$ 2$, we call the obstructions to embedding and isotopy, respectively. We let $\pi_{i}$ denote the sheaf of homotopy groups $\pi_{i}\left(\pi_{M}{ }^{\prime}\right)$ for each integer $i \geqq 1$; the first obstruction to finding an embedding of $V$ in $M$ homotopic to $f$ lies in $H^{n}\left(\boldsymbol{R}^{*} V ; \pi_{n-1}\right)$; higher obstructions lie in $H^{n+i}\left(R^{*} V ; \pi_{n+i-1}\right)$ for $i \geqq 1$. The first obstruction to finding an isotopy of $\left\{f_{t}\right\}$ and $\left\{g_{t}\right\}$ (which can also be thought of as the first obstruction to finding an isotopy of $f_{1}$ with $g_{1}$ which is homotopic to $\left\{r_{t}\right\}$, where $r_{t}=f_{1-2 t}$ if $0 \leqq t \leqq \frac{1}{2}, r_{t}=$ $g_{2 t-1}$ if $\left.\frac{1}{2} \leqq t \leqq 1\right)$ lies in $H^{n-1}\left(\mathrm{R}^{*} V ; \pi_{n-1}\right)$; higher obstructions lie in $H^{n+i-1}\left(\mathrm{R}^{*} V ; \pi_{n+i-1}\right)$ for $i \geqq 1$.

3.3. The restatement of Haefliger's results.

THeOREM 3.3.1. Suppose $2 n \geqq 3(k+1)$. Then $f$ is homotopic to an embedding if and only if $\pi_{M}{ }^{\prime}$ has a section. Furthermore, if $\Phi$ is a section of $\pi_{M}{ }^{\prime}, f$ has an e-homotopy $\left\{f_{t}\right\}$ such that $\boldsymbol{\Phi}\left[f_{t}\right]$ is homotopic to $\Phi$.

Proof. If $\left\{f_{t}\right\}$ is an $e$-homotopy of $f, \Phi\left[f_{t}\right]$ is the desired section. Suppose $\Phi:\left(\mathbf{R}^{*} V, P V\right) \rightarrow\left(Y^{\prime}, Z^{\prime}\right)$ is a section of $\pi_{M}{ }^{\prime}$. Consider the diagram

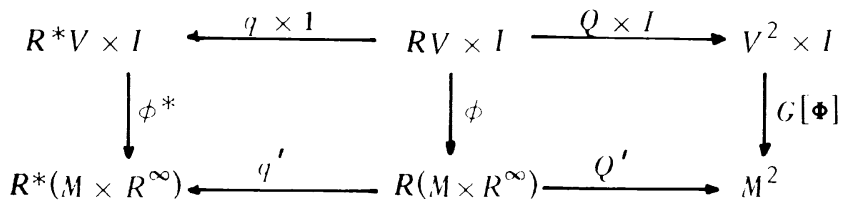

where $q$ and $q^{\prime}$ are the quotient maps, $Q=1 \cup \pi: R V \rightarrow V^{2}$, and $Q^{\prime}$ is the composition $\left(p_{1}\right)^{2} \circ(1 \cup \pi)$, where $p_{1}: M \times R^{\infty} \rightarrow M$ is projection to the first factor. The map $\phi^{*}$ is defined by $\phi^{*}(r, t)=\alpha(t)$ for all $(r, t) \in R^{*} V \times I$, where $\Phi(r)=(r, \alpha) \in Y^{\prime}$. G[ $\left.\Phi\right]$ and $\phi$ are the unique maps which make the diagram commute and which satisfy the equation $\phi(r, 0)=\boldsymbol{R}(f, i)(r)$ for all $r \in R V$. Now let $g_{t}: V_{2} \rightarrow M_{2}$, for all $0 \leqq t \leqq 1$, be the homotopy where $g_{t}(x, y)=G[\Phi](x, y, t)$ for all $(x, y) \in V^{2}$. Then $\left\{g_{t}\right\}$ is an equivariant homotopy; that is, $T \circ g_{t}$ $=g_{t} \circ T$ for all $t$, and $g_{1}$ is isovariant, i.e., $g_{1}{ }^{-1} \Delta_{M}=\Delta_{V}$. According 
to Theorem 1(a) of Haefliger [4], $f$ is homotopic to an embedding of $V$ into $M$.

Examining the details of Haefliger's proof, however, we observe that it is possible to construct an $e$-homotopy $\left\{f_{t}\right\}$ of $f$ and a 2-parameter homotopy $h_{\tau, t}: V^{2} \rightarrow M^{2}, 0 \leqq \tau, t \leqq 1$, such that $h_{0, t}=g_{t}$ and $h_{1, t}=f_{t}^{2}$ for all $t ; h_{\tau, 0}=g_{0}$ and $h_{\tau, 1}$ is isovariant for all $\tau$; and $h_{\tau, t}$ is equivariant for all $\tau, t$. Using $\left\{h_{\tau, t}\right\}$, we may show that $\Phi$ is homotopic to $\Phi\left[f_{t}\right]$; we leave the details to the reader.

THEOREM 3.3.2. Suppose $2 n>3(k+1)$. Then two e-homotupies of $f,\left\{f_{t}\right\}$ and $\left\{g_{t}\right\}$, are isotopic if and only if $\Phi\left[f_{t}\right]$ is homotopic to $\Phi\left[g_{t}\right]$.

Proof. If $\left\{f_{\tau, t}\right\}$ is an isotopy of $\left\{f_{t}\right\}$ with $\left\{g_{t}\right\}$, then $\left\{\Phi\left[f_{\tau, t}\right]\right\}_{0 \leqq \tau} \leqq 1$ is a homotopy of $\boldsymbol{\Phi}\left[f_{t}\right]$ with $\boldsymbol{\Phi}\left[g_{t}\right]$. Conversely, suppose $\boldsymbol{\Phi}_{\tau}$, for $0 \leqq \tau \leqq 1$, is a homotopy of sections of $\pi_{M}{ }^{\prime}$ such that $\Phi_{0}=\Phi\left[f_{t}\right]$ and $\Phi_{1}=\Phi\left[g_{t}\right]$. For each $\tau$, let $G\left[\Phi_{\tau}\right]: V^{2} \times I \rightarrow M^{2}$ be the map as constructed in the proof of 3.3.1. Let $h_{\tau, t}: V^{2} \rightarrow M^{2}$, for $0 \leqq \tau \leqq 1$, be the 2-parameter homotopy where $h_{\tau, t}(x, y)=G\left[\Phi_{\tau}\right](x, y, t)$ for all $(x, y)$ $\in V^{2}$. Note that $h_{\tau, 0}=f^{2}$ and $h_{\tau, 1}$ is isovariant for all $\tau ; h_{0, t}=f_{2 t}{ }^{2}$ and $h_{1, t}=g_{2 t}$ for all $0 \leqq t \leqq \frac{1}{2}$, and $h_{0, t}=f_{1}{ }^{2}$ and $h_{1, t}=g_{1}{ }^{2}$ for all $\frac{1}{2} \leqq t \leqq 1$; and $h_{\tau, t}$ is equivariant for all $\tau, t$. Thus $h_{\tau, 1}$, for $0 \leqq \tau \leqq 1$, is an isovariant homotopy of $f_{1}{ }^{2}$ with $g_{1}{ }^{2}$ which is equivariantly homotopic, rel $f_{1}{ }^{2}$ and $g_{1}{ }^{2}$, to the homotopy $r_{\tau}{ }^{2}: V^{2} \rightarrow M^{2}, 0 \leqq \tau \leqq 1$, where $r_{\tau}=f_{1-2 \tau}$ if $0 \leqq \tau \leqq \frac{1}{2}, g_{2 \tau-1}$ if $\frac{1}{2} \leqq \tau \leqq 1$. Haefliger's construction [4, Theorem 1 (b)] then gives us an isotopy of $f_{1}$ with $g_{1}$ which is homotopic to $\left\{r_{\tau}\right\}$. The construction of the isotopy of $\left\{f_{t}\right\}$ with $\left\{g_{t}\right\}$ is routine, and left to the reader.

3.4. The structure of the sheaf $\pi_{n-1}\left(\pi_{M}\right)$. In this paragraph, we insist that $n \geqq 2$.

Lemma 3.4.1. The inclusion $R\left(M \times R^{\infty}\right) \rightarrow\left(M \times R^{\infty}\right)^{2}$ is a homotopy equivalence.

Proof. Let $h_{t}: R^{\infty} \rightarrow R^{\infty}$, for $0 \leqq t \leqq 1$, be the isotopy where $h_{0}$ is the identity and where, for any integer $m \geqq 1$ and any $(m+1)^{-1}$ $\leqq t \leqq m^{-1}, h_{t}\left(x_{1}, x_{2}, \cdots\right)=\left(y_{1}, y_{2}, \cdots\right)$, where $y_{i}=x_{i}$ for all $1 \leqq i<m, \quad y_{i}=x_{i-1}$ for all $i>m+1$, and $y_{m}=x_{m} \cos \theta$ and $y_{m+1}=x_{m} \sin \theta$, where $\theta=\frac{1}{2} \pi\left(t\left(m^{2}+m\right)-m\right)$. Note that $h_{1}$ is a homeomorphism of $R^{\infty}$ to the hyperplane $H_{0}$ of all points in $R^{\infty}$ with first coordinate 0 . Let $g_{t}: R^{\infty} \rightarrow R^{\infty}$, for $0 \leqq t \leqq 1$, be the isotopy where $g_{t}\left(x_{1}, x_{2}, \cdots\right)=\left(x_{1}+t, x_{2}, \cdots\right)$, i.e., translation along the $x_{1}$-axis. We define a homotopy $r_{t}:\left(M \times R^{\infty}\right)^{2} \rightarrow\left(M \times R^{\infty}\right)^{2}$, $0 \leqq t \leqq 1$, as follows: 


$$
r_{t}(x, v, y, w)= \begin{cases}\left(x, h_{2 t} v, y, h_{2 t} w\right) & \text { if } 0 \leqq t \leqq \frac{1}{2} \\ \left(x, h_{1} v, y, g_{2 t-1} w\right) & \text { if } \frac{1}{2} \leqq t \leqq 1\end{cases}
$$

for all $x, y \in M$ and $v, w \in R^{x}$. Note that $r_{0}$ is the identity, $r_{1}\left(M \times R^{\infty}\right)^{2} \subset R\left(M \times R^{\infty}\right)$, and $r_{t}\left(R\left(M \times R^{x}\right)\right) \subset R\left(M \times R^{\infty}\right)$ for all $t$; thus $r_{1}$ is a homotopy inverse of the inclusion, and we are done.

Let $Q=1 \cup \pi: R M \rightarrow M^{2}$ be the quotient map, where $\pi: S M \rightarrow M$ $=\Delta_{M}$ is the projection. Let $e: R \rightarrow M^{2}$ be a fibration replacing $Q$, i.e., $R=\left\{(r, \alpha) \in R M \times\left(M^{2}\right)^{I} \mid Q \circ \alpha(1)=r\right\}$, and let $e(r, \alpha)=\alpha(0)$. Let $S=\left\{(r, \alpha) \in R \mid p_{1} \circ \alpha\right.$ is constant $\}$, where $p_{1}: M^{2} \rightarrow M$ is projection to the first factor. We pick a basepoint $x \in M$ and a local orientation of $M$ at $x$, which we represent by a homeomorphism $\omega: S^{n-1} \rightarrow S M_{x}, S M_{x}$ being the set of unit tangents of $M$ at $x$. For each loop $\sigma$ of $M$, we define a map $\chi[\sigma]: S^{n-1} \rightarrow R_{x}$, where $R_{x}=e^{-1}(x, x), \quad$ as follows: $\quad \chi[\sigma](v)=(\omega(v), \alpha)$, where $\alpha(t)=$ $(x, \alpha(t))$, for all $v \in S^{n-1}$. The homotopy class of $\chi[\sigma]$ clearly depends only on the homotopy class of $\sigma$, hence if $[\sigma]=g \in \pi_{1}(M, x)$, we define $X(\mathrm{~g})$ to be the homotopy class of $\chi[\sigma]$.

LemMA 3.4.2. As an Abelian group, $\pi_{n-1}\left(R_{\mathrm{x}}\right)$ is freely generated by the set of all $X(g)$, for $g \in \pi_{1}(M, x)$.

Proof. Consider the commutative diagram

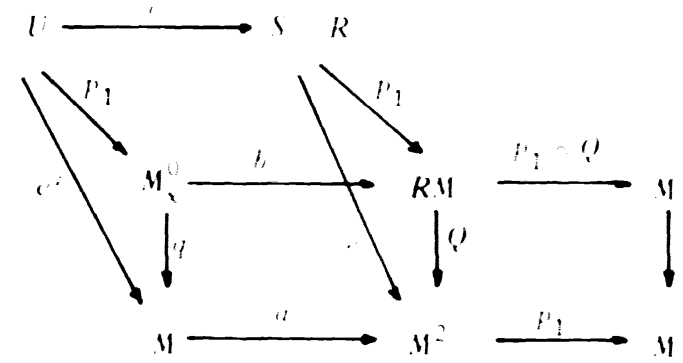

where $a y=(x, y)$ for all $y \in M, M_{x}{ }^{0}=(M-\{x\}) \cup S M_{x}$, with the topology which makes $b=a \cup \pi$ an embedding, where $U=\{(r, \alpha) \in$ $\left.M_{x}{ }^{\prime \prime} \times M^{I} \mid q \circ \alpha(1)=r\right\}, \quad$ and $\quad p_{1}(r, \alpha)=r, \quad e(r, \alpha)=\alpha(0), \quad$ and $c(r, \alpha)=(b r, \beta)$ for all $(r, \alpha) \in U$, where $\beta(t)=(x, \alpha(t))$ for all $t$. Now since $p_{1}: M^{2} \rightarrow M$ and $p_{1} \circ Q$ are both fibrations with fibers $M$ and $M_{x}{ }^{0}$, respectively, and since $p_{1}: U \rightarrow M_{x}{ }^{1}$ and $p_{1}: R \rightarrow R M$ are homotopy equivalences (as the reader can easily check), the inclusion $S \subset R$ is a homotopy equivalence. Also (where $U_{x}=\left(e^{\prime}\right)^{-1} x$ ), $c$ maps $U_{x}$ homeomorphically to $S_{x}=R_{x} \cap S$, which is of the homotopy type of $R_{x}$.

Let $\lambda: M \rightarrow M$ be a universal covering of $M$, and pick $x \in \lambda^{-1} x$. 
Let $M_{x}{ }^{0}$ and $U$ be the pullbacks, as in the diagram

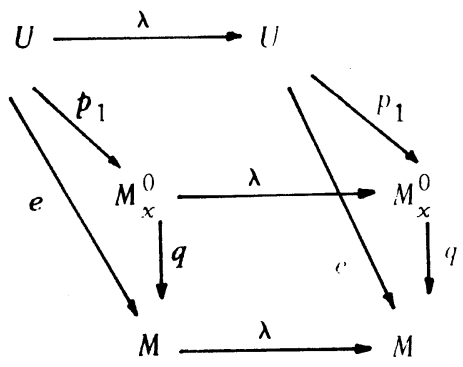

$M$ is simply connected. By a Serre spectral sequence argument, we can show that the fiber of $\boldsymbol{e}$ (hence also that of $e$ ) is of the homotopy type, through dimension $n-1$, of the loop space of the cofiber of $q$, which is a wedge of $n$-spheres, one for each element of $\pi_{1}(M, x)$. We leave the remaining details to the reader.

LEMMA 3.4.3. If $\pi_{r}(M)=0$ for all $1<r \leqq m$ for some integer $2 \leqq m \leqq n$, then $\pi_{n+m-2}\left(R_{x}\right)$ is isomorphic to $\pi_{n-1}\left(R_{x}\right) \otimes \pi_{m-1}$, where $\pi_{m-1}$ is the stable $(m-1)$-stem in the homotopy of spheres.

Proof. Let $\pi_{n-1}\left(R_{x}\right) \otimes \pi_{n-1} \rightarrow \pi_{n+m-2}\left(R_{x}\right)$ be the homomorphism which sends each $g \otimes h$ to $g \circ h$. We refer the reader to the proof of Theorem 3.4.2 above. Since $M$ is $m$-connected, $R_{x}$ has the homotopy type of the loop space of a wedge of $n$-spheres up through dimension $n+m-2$; we omit the details.

We henceforth express elements of $\pi_{n-1}\left(R_{x}\right)$ as formal sums of the $X(g)$ for values of $g \in \pi_{1}(M, x)$.

Let $\mu: \pi_{n-1}\left(R_{x}\right) \times \pi_{1}\left(M^{2},(x, x)\right) \rightarrow \pi_{n-1}\left(R_{x}\right)$ be the usual (right) action of the fundamental group of a base on the homotopy of a fiber. We shall identify $\pi_{1}\left(M^{2},(x, x)\right)$ with $\pi_{1}(M, x) \oplus \pi_{1}(M, x)$ in the usual way.

LemMA 3.4.4. If $g, h \in \pi_{1}(M, x)$, then $\mu(\chi(g),(h, 1))=\chi\left(h^{-1} g\right)$, where $1 \in \pi_{1}(M, x)$ is the identity.

Proof. Let $\sigma$ be a loop in $M$ which represents $g$, and $\tau$ a loop which represents $h$. Consider a map $\nu: S^{n-1} \times I \rightarrow S$ defined as follows: $\nu(v, t)=(\omega(v), \alpha)$ for all $v \in S^{n-1}$ and all $t \in I$, where

$$
\alpha(u)= \begin{cases}(x, \tau(-(t+1) u+t)) & \text { if } 0 \leqq u \leqq t /(t+1) \\ (x, \sigma((t+1) u-t)) & \text { if } t /(t+1) \leqq u \leqq 1\end{cases}
$$

Note that $[\nu(, 0)]=\chi(g), \quad[\nu(, 1)]=\chi\left(h^{-1} g\right), \quad$ and $\quad[\nu(, t)] \in$ $\pi_{n-1}\left(e^{-1}(\tau(t), x)\right)$ for all $t \in I$, and we are done. 
LEMMA 3.4.5. If $g, h \in \pi_{1}(M, x), \mu(\chi(g),(1, h))=(-1)^{d(h)} \chi(g h)$, where $d$ is the orientation homomorphism (cf. 1.2) and $\mu$ is the usual action of the fundamental group of the base on the homotopy of the fiber.

Proof. Let $\sigma$ and $\tau$ be loops in $M$ representing $g$ and $h$, respectively. Let $\omega_{t}: S^{n-1} \rightarrow S M_{\tau(t)}$, for $0 \leqq t \leqq 1$, be a homotopy such that $\omega_{0}=\omega$. Note then that $\omega_{1}=\omega \circ \epsilon$, where $\epsilon: S^{n-1} \rightarrow S^{n-1}$ is a map of degree $(-1)^{d(h)}$. We define $\xi: S^{n-1} \times I \rightarrow S$ as follows: $\xi(v, t)=\left(\omega_{t}(v), \alpha\right)$ for all $v \in S^{n-1}$ and $t \in I$, where

$$
\alpha(u)= \begin{cases}(x, \sigma((1+t) u)) & \text { if } 0 \leqq u \leqq(1+t)^{-1} \\ (x, \tau((1+t) u-1)) & \text { if }(1+t)^{-1} \leqq u \leqq 1\end{cases}
$$

Our proof is complete, since $[\xi(, 0)]=\chi(g)$.

$$
[\xi(, 1)]=\chi(g h)[\epsilon]=(-1)^{d(h)} \chi(g h),
$$

and

$$
[\xi(, t)] \in \pi_{n-1}\left(e^{-1}(x, \tau(t))\right) \quad \text { for all } t \in I .
$$

As before, let $T: M^{2} \rightarrow M^{2}$ and $T: R M \rightarrow R M$ be as defined in $\$ 3.1$. Let $T$ operate on the path space $\left(M^{2}\right)^{I}$ by composition. Now $R$ is an invariant subspace of $R M \times\left(M^{2}\right)^{I}$ under $T$, but $S$ is not.

LEMma 3.4.6. If $g \in \pi_{1}(M, x), T_{*} X(g)=(-1)^{n}(-1)^{d(g} \chi\left(g^{-1}\right)$.

Proof. Let $\tau$ be a loop in $M$ which represents $g$, and let $\omega_{t}: S^{n-1}$ $\rightarrow S M_{\tau(t)}$ be the homotopy as defined in the proof of Lemma 3.4.5 above. Let $\zeta: S^{n-1} \times I \rightarrow R$ be the homotopy where, for all $v \in S^{n-1}$ and $t \in I, \zeta(v, t)=\left(\omega_{t}, \alpha\right)$, where $\alpha(u)=(\tau(u t), \tau(1-u+u t))$ for all $0 \leqq u \leqq 1$. Now $\zeta(v, t) \in R_{x}$ for all $(v, t)$; thus $[\zeta(, 1)]=$ $[\zeta(, 0)]=\chi(g)$, while

$$
\begin{aligned}
{[T \circ \zeta(, 1)] } & =\chi\left(g^{-1}\right)[\epsilon] \\
& =(-1)^{n}(-1)^{d(g)} \chi\left(g^{-1}\right),
\end{aligned}
$$

where $\epsilon=T \circ \omega_{1} \circ \omega_{0}^{-1}, T$ being the antipodal map on $S^{n-1}$. We are done.

Let $Q^{\prime}=1 \cup \pi: \boldsymbol{R}\left(M \times R^{\infty}\right) \rightarrow\left(M \times R^{\infty}\right)^{2}$ be the quotient map. Let $W=\left\{(r, r) \in R \times R\left(M \times R^{\infty}\right) \mid e(r)=\left(p_{1}{ }^{2} \circ Q^{\prime}\right) r\right\}$. Since $T$ acts on $R, M^{2}$, and $\boldsymbol{R}\left(M \times R^{\infty}\right)$, and $T \circ e=e \circ T$ and $T \circ p_{1}{ }^{2} \circ \dot{Q}^{\prime}$ $=p_{1}{ }^{2} \circ Q^{\prime} \circ T, T$ also acts on $W$. Let $W^{*}=W / T$. Consider diagram (3.4-1) below, in which $W$ is the pullback: 


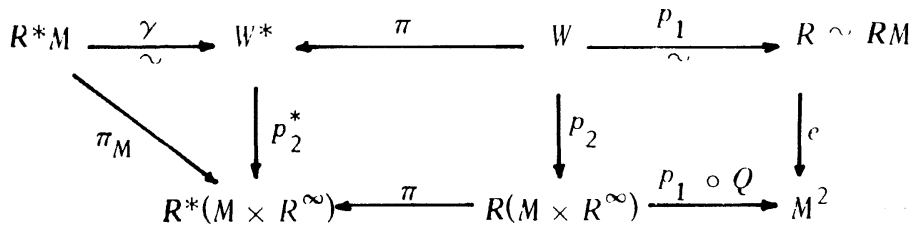

where, for any $\alpha \in Y$, i.e., $\alpha: I \rightarrow R^{*}\left(M \times R^{\infty}\right)$ and $\alpha(1) \in R^{*} M$, we let $\boldsymbol{\alpha}: I \rightarrow \boldsymbol{R}\left(M \times R^{\infty}\right)$ be one of the two paths where $\pi \circ \boldsymbol{\alpha}=\alpha$; we then define $\gamma(\alpha)$ to be the unordered pair

$$
\left(\left(\boldsymbol{\alpha}(1), p_{1}{ }^{2} \circ Q^{\prime} \circ \boldsymbol{\alpha}\right), \boldsymbol{\alpha}(0)\right),\left(\left(T \circ \boldsymbol{\alpha}(1), T \circ p_{1}{ }^{2} \circ Q^{\prime} \circ \boldsymbol{\alpha}\right), \quad T \circ \boldsymbol{\alpha}(0)\right) \in W^{*} .
$$

Since by Lemma 3.4.1, $p_{1}{ }^{2} \circ Q^{\prime}$ is a homotopy equivalence, $\gamma$ is a homotopy equivalence.

Pick $v \in S\left(M \times R^{\infty}\right)$ to be a unit vector at $(x, 0) \in M \times R^{\infty}$. Let $v^{*}=\{v,-v\} \in P\left(M \times R^{\infty}\right)$, and let $Y_{v}=\pi_{M}{ }^{-1} v^{*} \subset Y$. Let $W_{v}=$ $\left(p_{2}\right)^{-1} v \subset W$ and $W_{v}{ }^{*}=\left(p_{2}{ }^{*}\right)^{-1} v^{*} \subset W^{*}$. Now $\left(p_{1} \circ \pi^{-1 \circ} \gamma\right)$ : $Y_{v} \rightarrow R_{x}$ is a homotopy equivalence; we define $Y(g)=$ $\left(p_{1} \circ \pi^{-1 \circ \gamma}\right)_{\#}-\mathrm{l} \chi(g)$ for all $g \in \pi_{1}(M, x) ; \pi_{n-1}\left(Y_{v}\right)$ is freely generated by the $Y(g)$.

Let $U_{v}=U \cap Y_{v}$. We let $\theta: S^{n-1} \times I \rightarrow S\left(M \times R^{\infty}\right)_{(x, 0)}$ be any map such that $\theta(w, 0)=v$ and $\theta(w, 1)=\omega(w)$ for all $w \in S^{n-1}$, and let $\eta: S^{n-1} \rightarrow U_{v}$ be the map where, for all $w \in S^{n-1}, \eta(w)=\alpha$ with $\alpha(t)=\pi \circ \theta(w, t)$ for all $0 \leqq t \leqq 1$, where $\pi: S\left(M \times R^{\infty}\right) \rightarrow P\left(M \times R^{\infty}\right)$ is the covering map. Since $S\left(M \times R^{\infty}\right)_{(x, 0)} \cong S^{\infty}, \theta$ exists and is unique up to homotopy rel $S^{n-1} \times \partial I$, hence $\eta$ exists and is unique up to homotopy. Let $\psi \in \pi_{n-1}\left(U_{v}\right)$ be the class containing $\eta$.

LEMMA 3.4.7. $\pi_{n-1}\left(U_{z}\right) \cong Z$ and is generated by $\psi$.

Proof. Let $U^{\prime}=\left\{\alpha \in P\left(M \times R^{\infty}\right)_{(x, 0)}^{I} \mid \alpha(1) \in P M_{x}\right\}$, and consider the commutative diagram

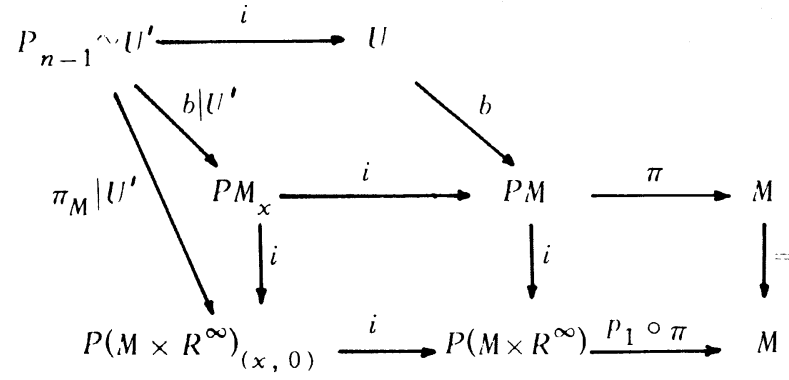

where each map labeled " $i$ " is an inclusion and $b(\alpha)=\alpha(1)$ for all 
$\alpha \in U$. Now $b$ and $b \mid U^{\prime}$ are both homotopy equivalences, $P M_{x}$ and $P\left(M \times R^{\infty}\right)_{(x, 0)}$ (which are, respectively, homeomorphic to real projective spaces $P_{n-1}$ and $\left.P_{\infty}\right)$ are the fibers of $\pi$ and $p_{1}{ }^{\circ} \pi$, respectively; thus the inclusion $U^{\prime} \subset U_{v}$ is a homotopy equivalence. $U^{\prime}$ is of the homotopy type of $S^{n-1}$, the fiber of the inclusion $P_{n-1} \subset P_{\infty}$; and $\eta: S^{n-1} \rightarrow U^{\prime}$ is a homotopy equivalence. The result follows.

THEOREM 3.4.8. (i) $\left(p_{1} \circ \pi^{-1} \circ \gamma\right)_{\#}: \pi_{n-1}\left(U_{v}\right) \rightarrow \pi_{n-1}\left(R_{x}\right)$ maps $\psi$ to $\chi(1)$, where $1 \in \pi_{1}(M, x)$ is the identity.

(ii) $\gamma_{\#}(\psi)=\Upsilon(1)$.

Proof. (i) We routinely verify that $p_{1} \circ \pi^{-1} \circ \gamma^{\circ} \eta=\chi[0]$, where 0 is the trivial loop at $x \in M$. Part (ii) follows immediately from (i).

Define functions $\rho: \pi_{1}\left(R^{*}\left(M \times R^{\infty}\right), \quad v^{*}\right) \rightarrow \pi_{1}(M, x) \otimes \pi_{1}(M, x)$ and $\delta: \pi_{1}\left(R^{*}\left(M \times R^{\infty}\right), v^{*}\right) \rightarrow Z_{2}$, as follows: If

$$
g \in \pi_{1}\left(\boldsymbol{R}^{*}\left(M \times R^{\infty}\right), v^{*}\right),
$$

pick a loop $\sigma$ representing $g$ and let $\tau$ be the path in $\boldsymbol{R}\left(M \times R^{\infty}\right)$ such that $\tau(0)=v$ and $\pi \circ \tau=\sigma$. Let $\delta(g)=0$ if $\tau(1)=v, 1$ if $\tau(1)=-v$. Now $Q^{\prime} v=Q^{\prime}(-v)=(x, 0)$, so $p_{1}{ }^{2} \circ Q^{\prime} \circ \tau$ is a loop in $M^{2}$; let $\rho(g)$ be the homotopy element represented by that loop.

We remark that $\delta$ is a homomorphism but $\rho$ is not; in fact, if $g, h \in \pi_{1}\left(R^{*}\left(M \times R^{\infty}\right), \quad v^{*}\right), \quad \rho(g h)=\rho(g)\left(T^{\delta(g)} \rho(h)\right), \quad$ where $T$ exchanges coordinates.

Let $G[M]$ be the local system of Abelian groups (i.e., locally trivial sheaf) over $\boldsymbol{R}^{*}\left(M \times R^{\infty}\right)$ such that for each $r \in R^{*}\left(M \times R^{\infty}\right), G[M]_{r}=$ $\pi_{n-1}\left(\pi_{M^{-1}} r\right)$. Let $G=G[M]_{v^{*}}$, and let $\mu: G \times \pi_{1}\left(\boldsymbol{R}^{*}\left(M \times R^{\infty}\right), v^{*}\right)$ $\rightarrow G$ be the usual (right) action of the fundamental group of a space on the stalk of a local system at the basepoint. We summarize the results of $\$ 3.4$ in the following theorem.

TheOREM 3.4.9. (i) $G$ is freely generated by $\left\{\Upsilon(g) \mid g \in \pi_{1}(M, x)\right\}$. (ii) If $g \in \pi_{1}(M, x)$ and $h \in \pi_{1}\left(\boldsymbol{R}^{*}\left(M \times R^{\infty}\right)\right.$, $\left.v^{*}\right)$, let $\rho(h)=\left(h_{1}, h_{2}\right)$. Then

$$
\mu(\Upsilon(g), h)= \begin{cases}(-1)^{d\left(h_{2}\right.} \Upsilon\left(h_{1}^{-1} g h_{2}\right) & \text { if } \delta(h)=0 \\ (-1)^{d\left(g h_{2}\right)}(-1)^{n} \Upsilon\left(h_{2}^{-1} g^{-1} h_{1}\right) & \text { if } \delta(h)=1\end{cases}
$$

(iii) $\pi_{n-1}\left(\pi_{M}\right)$ is the unique subsheaf of $G[M]$ such that $\pi_{n-1}\left(\pi_{M}\right)_{r}$ $=G[M]_{r}$ if $r \notin P\left(M \times R^{\infty}\right), \quad \pi_{n-1}\left(\pi_{M}\right)_{v^{*}}$ is the subgroup of $G$ generated by $Y(1)$, and $\pi_{n-1}\left(\pi_{M}\right) \mid P\left(M \times R^{\infty}\right)$ is locally trivial, i.e., locally a product sheaf (isomorphic to $Z$ ), provided $M$ is connected. (iv) If, for some integer $2 \leqq r \leqq n-2, \pi_{i}(M, x)=0$ for all $2 \leqq i \leqq r$, 
$\pi_{n+r-2}\left(\pi_{M}\right) \cong \pi_{n-1}\left(\pi_{M}\right) \otimes \pi_{r-1}$, where $\pi_{r-1}$ is the stable $(r-1)$-stem in the homotopy of spheres.

3.5. Action of $\pi_{1}\left(M^{v}, f\right)$. Let us reconsider diagram (3.2-1). Suppose that $\left\{f_{t}\right\}$ is a differentiable self-homotopy of $f$, i.e., $f_{0}=$ $f_{1}=f$, and each $f_{t}$ is differentiable. We define a map of pairs $\Gamma\left[f_{t}\right]:\left(Y^{\prime}, Z^{\prime}\right) \rightarrow\left(Y^{\prime}, Z^{\prime}\right)$ such that $\pi_{M}{ }^{\prime} \circ \Gamma\left[f_{t}\right]=\pi_{M}{ }^{\prime}$ as follows. If $(r, \alpha) \in Y^{\prime}$, where $r \in R^{*} V$ and $\alpha: I \rightarrow R^{*}\left(M \times R^{\infty}\right)$ is a path such that $\boldsymbol{\alpha}(0)=\boldsymbol{R}^{*}(f, i)(r)$ and $\boldsymbol{\alpha}(1) \in \boldsymbol{R}^{*} M \quad$ (cf. \$3.2), let $\Gamma\left[f_{t}\right](r, \alpha)=$ $(r, \beta)$, where $\beta(t)=R^{*}\left(f_{1-2 t}, i\right)(r)$ if $0 \leqq t \leqq \frac{1}{2}$ and $\beta(t)=\alpha(2 t-1)$ if $\frac{1}{2} \leqq t \leqq 1$.

We say that two maps $\Gamma_{0}, \Gamma_{1}:\left(Y^{\prime}, Z^{\prime}\right) \rightarrow\left(Y^{\prime}, Z^{\prime}\right)$ such that $\pi_{M}{ }^{\prime} \circ \Gamma_{i}$ $=\pi_{M}{ }^{\prime}$ for $i=0,1$ are homotopic if we can find a homotopy $\Gamma_{t}$ : $\left(Y^{\prime}, Z^{\prime}\right) \rightarrow\left(Y^{\prime}, Z^{\prime}\right)$, for $0 \leqq t \leqq 1$, such that $\pi_{M}{ }^{\prime} \circ \Gamma_{t}=\pi_{M}{ }^{\prime}$ for all $t$. The proofs of the following remarks are routine homotopy arguments, which we omit.

Remark 3.5.1. If $\left\{f_{t}{ }^{\prime}\right\}$ is another differentiable self-homotopy of $f$ which is homotopic to $\left\{f_{t}\right\}$ rel $f, \Gamma\left[f_{t}^{\prime}\right]$ is homotopic to $\Gamma\left[f_{t}\right]$.

REMARK 3.5.2. If $\left\{g_{t}\right\}$ is another differentiable self-homotopy of $f$ and if $\left\{h_{t}\right\}$ is the self-homotopy such that $h_{t}=f_{2 t}$ if $0 \leqq t \leqq \frac{1}{2}$ and $h_{t}=g_{2 t-1}$ if $\frac{1}{2} \leqq t \leqq 1$, then $\Gamma\left[h_{t}\right]$ is homotopic to $\Gamma\left[g_{t}\right] \circ \Gamma\left[f_{t}\right]$.

REMARK 3.5.3. If $f_{t}=f$ for all $t$, then $\Gamma\left[f_{t}\right]$ is homotopic to the identity.

We can thus define a right action $\gamma: \operatorname{Sec}\left(\pi_{M}{ }^{\prime}\right) \times \pi_{1}\left(M^{V}, f\right) \rightarrow$ $\operatorname{Sec}\left(\pi_{M}{ }^{\prime}\right)$ as follows: $\gamma\left([c],\left[f_{t}\right]\right)=\left[\Gamma\left[f_{t}\right] \circ c\right]$ for any section $c$ of $\pi_{M}{ }^{\prime}$ and any differentiable self-homotopy $\left\{f_{t}\right\}$ of $f$, where $\left[f_{t}\right]$ is the corresponding element of the fundamental group of $M^{V}$. Let $\pi_{i}=\pi_{i}\left(\pi_{M}{ }^{\prime}\right)$ for any integer $i \geqq 1$. We have a right action of $\pi_{1}\left(M^{V}, f\right)$ on the sheaf $\pi_{i}$, namely $\gamma_{*}: \pi_{i} \times \pi_{1}\left(M^{V}, f\right) \rightarrow \pi_{i}$ where, for any $r \in \boldsymbol{R}^{*} V$ and $g=\left[f_{t}\right] \in \pi_{1}\left(M^{V}, f\right), \gamma_{*}(, g)$ is the automorphism $\Gamma\left[f_{t}\right]_{\#}$ on the stalk $\pi_{i}\left(\pi_{M}{ }^{\prime}\right)_{r}$. We also let $\gamma_{*}: H^{*}\left(\boldsymbol{R}^{*} V ; \pi_{i}\right) \times \pi_{1}\left(M^{V}, f\right) \rightarrow$ $H^{*}\left(\boldsymbol{R}^{*} V ; \pi_{i}\right)$ be the action obtained by applying $\gamma_{*}$ to the coefficient sheaf.

The following remark follows immediately from a simple naturality argument:

Remark 3.5.4. If $g \in \pi_{1}\left(M^{v}, f\right)$ and if $c_{0}, c_{1}$ are sections of $\pi_{M}{ }^{\prime}$ over $\left(R^{*} V\right)^{m}$, the $m$-skeleton of $R^{*} V$, for some $m \geqq 0$, and if $h_{\tau}$ for $0 \leqq \tau \leqq 1$ is a homotopy of sections of $\pi_{M}{ }^{\prime}$ over $\left(R^{*} V\right)^{m-1}$ such that $h_{i}=$ $c_{i} \mid\left(R^{*} V\right)^{m-1}$ for $i=0$ and 1 , then

$$
\gamma^{*}\left(d^{m}\left(c_{0}, c_{1} ; h_{\tau}\right), g\right)=d^{m}\left(\Gamma\left[f_{t}\right] \circ c_{0}, \Gamma\left[f_{t}\right] \circ c_{1} ; \Gamma\left[f_{t}\right] \circ h_{\tau}\right)
$$

where $\left\{f_{t}\right\}$ is any self-homotopy of $f$ which represents $g$. 
Now let $\gamma_{\#}:[V \subset M]_{f} \times \pi_{1}\left(M^{V}, f\right) \rightarrow[V \subset M]_{f}$ be the right action defined as follows: If $\left\{g_{t}\right\}$ is any differentiable self-homotopy of $f$ and if $\left\{f_{t}\right\}$ is any $e$-homotopy of $f$, let $\gamma_{\#}\left(\left[f_{t}\right],\left[g_{t}\right]\right)=\left[h_{t}\right]$, where $\left\{h_{t}\right\}$ is the $e$-homotopy: $h_{t}=g_{1-2 t}$ if $0 \leqq t \leqq \frac{1}{2}, h_{t}=$ $f_{2 t-1}$ if $\frac{1}{2} \leqq t \leqq 1$. The actions $\gamma_{*}$ and $\gamma_{\#}$ are consistent, i.e., if $\boldsymbol{\phi}:[V \subset M]_{f} \rightarrow \operatorname{Sec}\left(\pi_{M}{ }^{\prime}\right)$ is the function defined in $\$ 1.1, \gamma \neq(\phi e, g)=$ $\phi\left(\gamma_{*}(e, g)\right)$ for all $e \in[V \subset M]_{f}, g \in \pi_{1}\left(M^{V}, f\right)$.

Definition 3.5.1. Let $G$ be a group and $A$ an Abelian group. We say a function $\alpha: A \times G \rightarrow A$ is a right affine action of $G$ on $A$ if

(i) for all $a \in A$ and $g, h \in G, \alpha(a, g h)=\alpha(\alpha(a, g), h)$;

(ii) for all $a \in A, \alpha(a, 1)=a$, where $1 \in G$ is the identity;

(iii) for all $a, b \in A$ and $g \in G, \alpha(a+b, g)=\alpha(a, g)+\alpha(b, g)-$ $\alpha(0, g)$.

Suppose now that $k \geqq 2$ and $n=2 k+1$, and $f$ is an embedding. By Theorem 2.5.1, we may identify $[V \subset M]_{f}$ with $H^{2 k}\left(\boldsymbol{R}^{*} V ; \pi_{2 k}\right)$, where $[f]$ corresponds to 0 . The following theorem follows immediately from 2.5.1 and 3.5.4:

THEOREM 3.5.5. If $f$ is an embedding and $n=2 k+1$, then $\gamma_{\#}:[M \subset V]_{f} \times \pi_{1}\left(M^{V}, f\right) \rightarrow[M \subset V]_{f}$ is a right affine action.

In general (without any dimensional restriction on $V$ and $M$ ) let $\Delta:[V \subset M]_{f} \rightarrow[V \subset M]$ be the function which takes $\left[f_{t}\right]$ to $\left[f_{1}\right]$ for each $e$-homotopy $\left[f_{t}\right]$ of $f$, as defined in $\$ 1.1$.

THeOREM 3.5.6. If $h: V \rightarrow M$ is an embedding homotopic to $f$, $\Delta^{-1}[h]$ is precisely an orbit of $[V \subset M]_{f}$ under the action $\gamma_{\#}$.

Proof. Choose an $e$-homotopy $\left\{f_{t}\right\}$ of $f$ such that $f_{1}=h$. Suppose that $\left\{g_{t}\right\}$ is a differentiable self-homotopy of $f$. Then $\boldsymbol{\gamma}_{\#}\left(\left[f_{t}\right],\left[g_{t}\right]\right)$ $=\left[k_{t}\right]$, where $k_{t}=g_{1-2 t}$ if $0 \leqq t \leqq \frac{1}{2}$ and $k_{t}=f_{2 t-1}$ if $\frac{1}{2} \leqq t \leqq 1$. $\Delta\left[k_{t}\right]=\left[k_{1}\right]=[h]$. Conversely, suppose that $\left\{r_{t}\right\}$ is another $e$ homotopy of $f$ such that $r_{1}=h$. Let $\left\{s_{t}\right\}$ be the self-homotopy of $f$ where $s_{t}=r_{2 t}$ if $0 \leqq t \leqq \frac{1}{2}$ and $s_{t}=f_{2-2 t}$ if $\frac{1}{2} \leqq t \leqq 1$. Then $\gamma_{\#}\left(\left[f_{t}\right],\left[s_{t}\right]\right)=\left[r_{t}\right]$.

3.6. Embeddings of $S^{k}$ in $M^{2 k+1}$. Suppose now that $S^{k}$ is the $k$ sphere, for $k \geqq 2$, and that $M$ is a connected manifold of dimension $n=2 k+1$. The space $R S^{k}$ is of the homotopy type of $S^{k}$, while $R^{*} S^{k}$ has the homotopy type of real projective $k$-space, $P_{k}$.

Definition 3.6.1. If $\mathcal{G}$ is any sheaf over $\boldsymbol{R}^{*} S^{k}$, let $\mathcal{G}^{0} \subset \mathcal{G}$ be the subsheaf where $\mathcal{G}_{r}{ }^{0}=0$ if $r \notin P S^{k}$, and $\mathcal{G}_{r}{ }^{0}=\mathcal{G}_{r}$ if $r \notin P S^{k}$. We remark that $H^{*}\left(\boldsymbol{R}^{*} S^{k} ; \mathcal{G}^{0}\right)=H^{*}\left(\boldsymbol{R}^{*} S^{k}, P S^{k} ; \mathcal{G}\right)[2]$.

Definition 3.6.2. If $A$ is an Abelian group and $\phi: A \rightarrow A$ is an automorphism such that $\phi^{2}=1$, the identity, let $[A, \phi]$ be the sheaf 
over $\boldsymbol{R}^{*} \boldsymbol{S}^{k}$ obtained from the product sheaf $\boldsymbol{R} S^{k} \times A$ by identifying $(r, a)$ with $(T r, \phi a)$ for all $r \in R S^{k}$ and $a \in A$.

Let $E: Z \oplus Z \rightarrow Z \oplus Z$ be the "exchange" automorphism, i.e., $E(x, y)=(y, x)$ for all $x, y \in Z$.

Consider the sheaf $\pi_{n-1}=\pi_{n-1}\left(\pi_{M}{ }^{\prime}\right)=\left(R^{*}(f, i)\right)^{-1} \pi_{n-1}\left(\pi_{M}{ }^{\prime}\right)$ over $\boldsymbol{R}^{*} S^{k}$, where $f: S^{k} \rightarrow M$ is any differentiable map, and $i: S^{k}$ $\rightarrow M$ is any embedding. We shall assume that $S^{k}$ and $M$ have basepoints $s_{0}$ and $m_{0}$, respectively, and $f\left(s_{0}\right)=m_{0}$.

$\boldsymbol{R} \mathbf{S}^{k}$ is simply connected, so $\pi_{n-1}$ breaks up as a direct sum (cf. Theorem 3.4.9); in fact $\pi_{n-1} \cong Z \oplus \sum_{g \neq 1} Z^{0}$, where $Z$ is the trivial integer sheaf, and the sum is over all $g \in \pi_{1}=\pi_{1}\left(M, m_{0}\right)$ not equal to the identity. We define sets $A \subset \pi_{1}$ and $B \subset \pi_{1}$ as follows: $A$ consists of all $g \in \pi_{1}$ such that $g \neq 1, g^{2}=1$, and $d(g)=0$, and $B$ consists of all $g \in \pi_{1}$ such that $g^{2}=1$ and $d(g)=1$, where $d: \pi_{1} \rightarrow$ $Z_{2}$ is the orientation homomorphism. Let $\Theta$ and $\Lambda$ be the sets of unordered pairs in $\pi_{1}$ as follows: $\Theta$ consists of all unordered pairs $\left\{g, g^{-1}\right\}$ such that $g^{2} \neq 1$ and $d(g)=0$, and $\Lambda$ consists of all $\left\{g, g^{-1}\right\}$ such that $g^{2} \neq 1$ and $d(g)=1$. Using the action of $\pi_{1}\left(\boldsymbol{R}^{*} S^{k}\right) \cong Z_{2}$ on the stalk of $\pi_{n-1}$, we obtain directly, from Theorem 3.4.9,

$$
\begin{aligned}
& \text { LEMMA 3.6.1. } \pi_{n-1} \cong[Z,-1] \oplus \sum_{A}[Z,-1]^{0} \oplus \sum_{B} Z^{0} \oplus \\
& \sum_{\Theta}[Z \oplus Z,-E]^{0} \oplus \sum_{\Lambda}[Z \oplus Z, E]^{0} .
\end{aligned}
$$

It is sufficient to compute the cohomology of $\boldsymbol{R}^{*} S^{k}$ with coefficients in each of the direct summands.

LEMMA 3.6.2. $H^{2 k}\left(\boldsymbol{R}^{*} S^{k} ;[Z,-1]\right)=0$.

Proof. $\boldsymbol{R}^{*} \boldsymbol{S}^{k}$ is of the homotopy type of a complex of dimension $k<2 k$, and $[Z,-1]$ is a local system.

LEMMA 3.6.3. $H^{2 k}\left(R^{*} S^{k} ;[Z,-1]^{0}\right)$ is isomorphic to $Z$ if $k$ is odd. $Z_{2}$ if $k$ is even.

Proof. $H^{2 k}\left(\boldsymbol{R}^{*} S^{k} ; \quad[Z,-1]^{0}\right)=H^{2 k}\left(\boldsymbol{R}^{*} S^{k}, P S^{k} ; \quad[Z,-1]\right)$. Now $R^{*} S^{k}$ is a $2 k$-manifold with boundary $P S^{k}$, which is oriented if $k$ is even and unoriented if $k$ is odd. In the even case, the generator of $H^{2 k}\left(\boldsymbol{R}^{*} S^{k} ;[Z,-1]^{0}\right)$ may be taken to be the top class.

LEMMA 3.6.4. $H^{2 k}\left(R^{*} S^{k} ; Z^{0}\right)$ is isomorphic to $Z$ if $k$ is even, $Z_{2}$ if $k$ is odd.

Proof. The proof is similar to that of Lemma 3.6.3, above. We leave the details to the reader.

LEMMA 3.6.5. $H^{2 k}\left(\boldsymbol{R}^{*} S^{k} ;[Z \oplus Z, E]^{0}\right) \cong Z$. 
Proof. We consider two cases; $k$ even and $k$ odd. We have exact sequences of sheaves

$$
\begin{aligned}
e_{1}: 0 \rightarrow Z=[Z, 1] \stackrel{\alpha}{\rightarrow}[Z \oplus Z, E] \stackrel{\beta}{\rightarrow}[Z,-1] \rightarrow 0, \\
e_{2}: 0 \rightarrow[Z,-1] \stackrel{\alpha}{\rightarrow}[Z \oplus Z, E] \stackrel{\leftrightarrow}{\rightarrow} Z=[Z, 1] \rightarrow 0,
\end{aligned}
$$

where the maps $\alpha, \beta, \gamma$, and $\epsilon$ can be defined on the underlying groups as follows: $\alpha x=(x, x)$ and $\gamma x=(x,-x)$ for all $x \in Z$, and $\beta(x, y)=x-y$ and $\epsilon(x, y)=x+y$ for all $x, y \in Z$. (Note that $\alpha, \beta, \gamma$, and $\epsilon$ all respect the appropriate actions; i.e., $E \circ \gamma=$ $\gamma \circ(-1)$, etc.) Note that $\epsilon \circ \boldsymbol{\alpha}$ is multiplication by 2 . Corresponding to $e_{1}$ and $e_{2}$, we have exact sequences in cohomology, where $\delta_{1}$ and $\delta_{2}$ are the Bokstein homomorphisms

$$
\begin{aligned}
& \left(e_{1}\right)_{*}: \stackrel{\delta_{1}}{\rightarrow} Z \stackrel{\alpha_{*}}{\rightarrow} H^{2 k}\left(\boldsymbol{R}^{*} S^{k}, P S^{k} ;[Z \oplus Z, E]\right) \stackrel{\beta_{*}}{\rightarrow} Z_{2} \rightarrow 0, \\
& \left(e_{2}\right)_{*}: \stackrel{\delta_{3}}{\rightarrow} Z_{2} \stackrel{\gamma_{*}}{\rightarrow} H^{2 k}\left(\boldsymbol{R}^{*} S^{k}, P S^{k} ;[Z \oplus Z, E]\right) \stackrel{\epsilon_{*}}{\rightarrow} Z \rightarrow 0,
\end{aligned}
$$

where $\epsilon_{*}{ }^{\circ} \alpha_{*}$ is multiplication by 2 . General algebraic considerations show that $\epsilon_{*}$ must be an isomorphism, and we are done. If $k$ is odd, the proof is the same with the roles of the sequences $e_{1}$ and $e_{2}$ reversed.

LEMMA 3.6.6. $H^{2 k}\left(\boldsymbol{R}^{*} S^{k} ;[Z \oplus Z,-E]^{0}\right) \cong Z$.

Proof. Analogous to $e_{1}$ and $e_{2}$ in the proof of Lemma 3.6.5, above, $[Z \oplus Z,-E]$ may be expressed both as an extension of $Z$ by $[Z,-1]$ and as an extension of $[Z,-1]$ by $Z$. We proceed as above.

From Lemmas 3.6.1 through 3.6.6, we immediately obtain

THEOREM 3.6.7. $\left[S^{k} \subset M\right]_{f}$ is isomorphic to $\sum_{A} Z \oplus \sum_{B} Z_{2} \oplus \sum_{\Theta \cup \Lambda} Z$ if $k$ is odd, and to $\sum_{A} Z_{2} \oplus \sum_{B} Z \oplus \sum_{\Theta \cup \Lambda} Z$ if $k$ is even.

3.7. Explicit geometric construction of $\left[S^{k} \subset M\right]_{f}$. We retain the notation of $\$ 3.5$, and assume that $f: S^{k} \rightarrow M$ is an embedding, where $f\left(s_{0}\right)=x ; s_{0}$ is the basepoint of $S^{k}$. Recall that we let $v \in S\left(M \times R^{\infty}\right)$ such that $\pi v=(x, 0)$. We can insist that $i: S^{k} \rightarrow R$ be an embedding where $\boldsymbol{i}\left(s_{0}\right)=0$.

Let $\sigma$ be a $2 k$-cell of $\boldsymbol{R}^{*} S^{k}$ such that, for some $w^{*} \in P S^{k}, w^{*} \in \partial \sigma$ and $\boldsymbol{R}^{*}(f, i)\left(w^{*}\right)=v^{*}=\{v,-v\} \in P\left(M \times R^{\infty}\right)$. Pick a cell $\tau \subset \boldsymbol{R S}^{k}$ such that $\pi \tau=\sigma$ and $w \in \partial \tau$ such that $\pi w=w^{*}$. Choose any ordered pair $\left(s_{1}, s_{2}\right) \in \operatorname{Int} \tau$, and let $N_{1}$ and $N_{2}$ be closed ball-shaped neighborhoods of $s_{1}$ and $s_{2}$, respectively, such that $N_{1} \times N_{1} \subset$ Int $\tau$. Let $\alpha: I \rightarrow \tau$ be a path such that $\alpha(0)=\left(s_{1}, s_{2}\right), \alpha(1)=w$, and $\alpha(t) \in$ Int $\tau$ for all $t<1$. Then, for all $0 \leqq t \leqq 1, \alpha(t)=\left(\alpha_{1}(t)\right.$, 
$\left.\alpha_{2}(t)\right)$, where $\alpha_{i}: I \rightarrow S^{k}$ is any path from $s_{i}$ to $s_{0}$, for $i=1$ and 2 . Pick any $g \in \pi_{1}=\pi_{1}(M, x)$. Let $\beta: I \rightarrow M$ be a simple smooth path such that $\beta(0)=f\left(s_{2}\right), \quad \beta(1)=f\left(s_{1}\right), \quad$ and the loop $\left(f \circ s_{2}{ }^{-1}\right)$ - $\beta\left(f \circ s_{1}^{-1}\right)$ represents $g$. Let $B$ be a neighborhood of $\beta(I)$ homeomorphic to a $(2 k+1)$-ball such that $B \cap f\left(S^{k}\right)=f\left(N_{1}\right) \cup f\left(N_{2}\right)$. Let $f_{t}: S^{k} \rightarrow M$, for $0 \leqq t \leqq 1$, be any homotopy of differentiable maps such that $f_{0}=f, f_{t}\left|\left(S^{k}-N_{2}\right)=f\right|\left(S^{k}-N_{2}\right)$ for all $t$, and $f_{t}\left(N_{2}\right) \subset B$ for all $t$, and where the map $F: S^{k} \times I \rightarrow M \times I$, where $F(s, t)=f_{t}(s)$ for all $s \in S^{k}$ and $0 \leqq t \leqq 1$, has just one double point; namely $F\left(s_{1}, \frac{1}{2}\right)=F\left(s_{2}, \frac{1}{2}\right)$, and $F\left(S^{k} \times I\right)$ meets itself transversely at $\left(f\left(s_{1}\right), \frac{1}{2}\right)$.

The liftings $\boldsymbol{\Phi}\left[f_{t}\right]$ and $\boldsymbol{\Phi}[f]$ are certainly homotopic on the $(2 k-1)$-skeleton of $R^{*} S^{k}$; in fact we may define $g_{u}:\left(\left(R^{*} S^{k}\right)^{2 k-1}\right.$, $\left.P S^{k}\right) \rightarrow\left(Y^{\prime}, Z^{\prime}\right)$ for $0 \leqq u \leqq 1$, explicitly, using the homotopy $\left\{f_{t}\right\}$ (we omit the details; $\left\{g_{u}\right\}$ is essentially the $\boldsymbol{\Phi}$-construction (cf. $3.2)$ restricted to the $(2 k-1)$-skeleton). Now consider the difference class:

$$
d^{2 k}=d^{2 k}\left(\Phi[f], \Phi\left[f_{t}\right] ; g_{u}\right) \in C^{2 k}\left(\boldsymbol{R}^{*} S^{k} ; \pi_{2 k}\right) .
$$

We can identify the stalk of $\pi_{2 k}$ over $w^{*}$ with that of $\pi_{2 k}\left(\pi_{M}\right)$ over $v^{*}$, and we have

LEMMA 3.7.1. $d^{2 k}(\boldsymbol{\sigma})= \pm \Upsilon(g)$ and $d^{2 k}\left(\boldsymbol{\sigma}^{\prime}\right)=0$ for any $2 k$-cell $\sigma^{\prime} \neq \boldsymbol{\sigma}$. Furthermore, we may insist $d^{2 k}(\boldsymbol{\sigma})=\Upsilon(\mathrm{g})$, by redefining $\left\{f_{t}\right\}$ if necessary.

Proof. Using the $\Phi$-construction, we may extend the homotopy $\left\{g_{u}\right\}$ over $\sigma^{\prime}$ for any $\sigma^{\prime} \neq \sigma$, hence $d^{2 k}\left(\sigma^{\prime}\right)=0$. Now (cf. 2.4) $d^{2 k}(\sigma)$ is represented by a map $h: \partial(\sigma \times I) \rightarrow Y$ such that, for all $(a, t) \in \partial(\sigma \times I)$ and all $0 \leqq u \leqq 1$,

$$
h(a, t)= \begin{cases}\boldsymbol{R}^{*}\left(f_{2 t u}(a), i\right) & \text { if } 0 \leqq u \leqq \frac{1}{2}, \\ \boldsymbol{R}^{*}\left(f_{t}(a),(2-2 u) i\right) & \text { if } \frac{1}{2} \leqq u \leqq 1,\end{cases}
$$

whose composition with $p_{1} \circ \pi^{-1} \circ \gamma$ (as in diagram 3.4-1) is homotopic to $\pm \mathbf{Y}(g)$. The sign is ambiguous, because there are essentially two ways an $r$-manifold can intersect itself transversely in a $2 r$ manifold. Both ways are possible in this case, hence we are done.

We now define $\langle g\rangle \in\left[S^{k} \subset M\right]_{f}$ to be $\left[f_{t}\right]$, where $\left\{f_{t}\right\}$ is described above. Theorem 1.2.1 then follows immediately from Theorem 3.6.7.

3.8. Free isotopy classes. In this paragraph, we assume that $f: S^{k} \rightarrow M$ is a small embedding, i.e., $f\left(S^{k}\right)$ lies in a single chart of $M$. Again, we assume that $k \geqq 2$ and $n=2 k+1$. We now investi- 
gate the affine action of $\pi_{1}\left(M^{S^{k}}, f\right)$ on $\left[S^{k} \subset M\right]_{f}$. Let $s_{0} \in S^{k}$ and $m_{0} \in M$ be basepoints, and assume that $f$ is basepoint-preserving.

Definition 3.8.1. Let $\left\{f_{t}\right\}$ be a differentiable self-homotopy of $f$. We say that $\left\{f_{t}\right\}$ is small if $f_{t}\left(S^{k}\right)$ lies in a single chart of $M$ for each $t$, and we say that $\left\{f_{t}\right\}$ is large if $f_{t}\left(s_{0}\right)=m_{0}$ for all $t$.

We remark that the subsets $L_{f}$ and $S_{f}$ of $\pi_{1}\left(M^{s^{k}}, f\right)$ represented by large and small self-homotopies of $f$, respectively, are subgroups, and that $L_{f} \cong \pi_{k+1}\left(M, m_{0}\right)$ and $S_{f} \cong \pi_{1}\left(M, m_{0}\right) . \quad L_{f}$ is normal, and $\pi_{1}\left(M s^{k}, f\right)$ is a semidirect product of $L_{f}$ with $S_{f}$; we leave this fact as an exercise.

THEOREM 3.8.1. If $x \in \pi_{1}\left(M^{s^{k}}, f\right)$ is represented by a small selfhomotopy $\left\{f_{t}\right\}$, then $\gamma(\langle g\rangle, x)=(-1)^{d(h)}\left\langle h^{-1} g h\right\rangle$ for all $g \in \pi_{1}\left(M, m_{0}\right)$, where $h$ is the element of $\pi_{1}\left(M, m_{0}\right)$ represented by the loop $\left\{f_{t}\left(s_{0}\right)\right\}$.

Proof. $\langle g\rangle$ is represented by a homotopy which extends a pseudopod out from $f\left(S^{k}\right)$, around a loop $\sigma$ representing $g$, then linking $f\left(S^{k}\right)$ with linking number 1 . The action of $x$ drags the entire image $f\left(S^{k}\right)$ around the loop $\alpha$, where $\alpha(t)=f_{t}\left(s_{0}\right)$ for all $t$; the pseudopod is now forced to follow the loop $\boldsymbol{\alpha}^{-1} \boldsymbol{\sigma} \boldsymbol{\alpha}$ and link with linking number $(-1)^{d(h)}$.

THeOREM 3.8.2. If $x \in L_{f}$, then $\gamma(\langle g\rangle, x)=\langle g\rangle+\gamma(0, x)$ for all $g \in \pi_{1}\left(M, m_{0}\right)$.

Proof. Since $x$ is represented by a large self-homotopy $\left\{f_{t}\right\}$, we may assume that $\left\{f_{t}\right\}$ leaves a neighborhood of $s_{0}, N$, fixed; we can insist that $N=B \cap \bigcup_{t} f_{t}\left(S^{k}\right)$, where $B$ is the $(2 k+1)$-ball used to construct $\langle g\rangle$ in $\$ 3.7$. Our theorem follows, because the difference cochain may be evaluated separately on $N$ and $S^{k}-N$, and the results added.

Theorem 1.2.2 follows directly from Theorem 3.8.1; we may extend this result slightly, using 3.8.2, as follows:

THEOREM 3.8.3. If $f: S^{k} \rightarrow M$ is a basepoint-preserving small embedding, then the subset of $\left[S^{k} \subset M\right]$ consisting of those isotopy classes homotopic to $f$ can be put into one-to-one correspondence with the set of orbits of the cokernel of a homomorphism $\Xi: \pi_{k+1}\left(M, m_{0}\right) \rightarrow\left[S^{k} \subset M\right]_{f}$ by a right action of $\pi_{1}\left(M, m_{0}\right)$; provided $k \geqq 2$ and $\operatorname{dim} M=2 k+1$.

Proof. Let $\iota: \pi_{k+1}\left(M, m_{0}\right) \rightarrow \pi_{1}\left(M^{s^{k}}, f\right)$ be the monomorphism onto $L_{f}$ induced by the map $S^{k+1} \rightarrow \Omega S^{k}$, and let $\Xi$ be defined by: 
$\Xi(x)=\gamma(0, \imath(x))$. By Theorem 3.8.2, $\Xi$ is a homomorphism. We can easily check that the action of $S_{f} \cong \pi_{1}\left(M, m_{0}\right)$ on $\left[S^{k} \subset M\right]_{f}$ is consistent with the usual right action of the fundamental group of a space on a higher homotopy group, via $\Xi$. We leave the details to the reader.

\section{BibLIOGRAPHY}

1. J. C. Becker, Cohomology and the classification of liftings, Trans. Amer. Math. Soc. 133 (1968), 447-475. MR 38 \#5217.

2. G. E. Bredon, Sheaf theory, McGraw-Hill, New York, 1967. MR 36 \#4552.

3. D. D. J. Hacon, Embeddings of $\mathrm{S}^{p}$ in $\mathrm{S}^{1} \times \mathrm{S}^{q}$ in the metastable range, Topology 7 (1968), 1-10. MR 36 \#5953.

4. A. Haefliger, Plongements différentiables dans le domaine stable, Comment. Math. Helv. 37 (1962/63), 155-176. MR 28 \#625.

5. S. T. Hu, Homotopy theory, Pure and Appl. Math., vol. 8, Academic Press, New York, 1959. MR 21 \#5186.

6. L. L. Larmore, The first obstruction to embedding a 1-complex in a 2-manifold, Illinois J. Math. 14 (1970), 1-11. MR 40 \#4955.

7. A. Shapiro, Obstructions to the imbedding of a complex in a euclidean space. I. The first obstruction, Ann. of Math. (2) 66 (1957), 256-269. MR 19, 671.

8. W. T. Wu, A theory of imbedding, immersion and isotopy of polytopes in a euclidean space, Science Press, Peking, 1965. MR 35 \#6146.

California State College, Dominguez Hills, California 90246 
